\title{
O impacto da cultura inovadora nas micro e pequenas empresas do Programa Agentes Locais De Inovação em Santa Catarina
}

\author{
The impact of innovative culture on micro and small enterprises \\ of the Program Local Innovation Agents in Santa Catarina
}

\author{
Ronivaldo Steingraber ${ }^{\mathrm{I}}$
}

\begin{abstract}
RESUMO
A literatura especializada não separa as micro das pequenas empresas. Apesar do porte pequeno, as dimensões são diferentes. Este artigo apresenta dois objetivos que se complementam. O primeiro é analisar os resultados do ciclo inicial (zero) para o ciclo um das empresas participantes do programa ALISC do SEBRAE e CNPQ. O período inicial desta fase é em 2015 e termina em 2018 e os dados mostram o desempenho das empresas que estão em todas as regiões do estado por duas faixas de tamanho: micro e pequena empresa. Aproveitando esta separação, os dados são analisados e comparados em função destas duas faixas de tamanho. Os resultados encontrados mostram que os dois tamanhos apresentam semelhanças e poucas diferenças. Os resultados apurados mostram que as micro e pequenas empresas seguem uma estratégia de aproximação do mercado, com maior desempenho das dimensões Rede e de Relacionamento.
\end{abstract}

Palavras chave: Inovação; Micro e pequena empresa; Radar da inovação

\begin{abstract}
The specialized literature does not separate micro and small enterprises. Despite the small size, the dimensions are different. This article presents two objectives that complement each other. The first is to analyze the results of the initial cycle (zero) for the cycle one of companies participating in the program ALISC of SEBRAE and CNPQ. The initial period of this phase is in 2015 and ends in 2018, and the data show the performance of companies that are in all regions of the state by two size ranges: micro and small enterprise. Taking advantage of this separation, the data are analyzed and compared according to these two size ranges. The results show that the two sizes have similarities and few differences. The results show that micro and small companies follow a strategy of market approximation, with greater performance of the dimensions Network and Relationship.
\end{abstract}

Key Words: Innovation; Micro and small enterprises; Radar of innovation

I Professor do Departamento de Economia e Relações Internacionais da UFSC -

ronivaldo.steingraber@gmail.com 


\section{INTRODUÇÃO}

O avanço da globalização e a mudança dos paradigmas produtivos lançaram luz ao avanço das micro e pequenas empresas (MPEs) nas últimas décadas. Mais flexíveis e adaptadas à acirrada competição internacional, elas ainda respondem rapidamente às políticas públicas de emprego e renda.

Experiências de países desenvolvidos nos anos 70 e 80 foram rapidamente reproduzidas nos países em desenvolvimento a partir dos anos 90, visando o estímulo às pequenas empresas, ao empreendedorismo, espirito de inovação e formação de novos empregos e distribuição de renda.

Todavia, as políticas de incentivo as MPEs também são cercadas de dúvidas.

A formação de MPEs é acompanhada de uma alta taxa de mortalidade destas empresas. Os mecanismos de financiamento são altamente enviesados para isenções, o que mostra a possível ineficiência econômica destes empreendimentos (como por exemplo, a baixa escala), oriundos mais da necessidade de trabalhadores desempregados do que 0 avanço de empreendedores e de espírito inovador.

Por outro lado, a teoria ortodoxa pouco se debruça sobre este problema. A alta competição entre as pequenas empresas sempre foi vista como importante para o bom funcionamento do mercado. Outras teorias se dedicaram mais a análise das grandes empresas, que organizacionalmente são mais completas e não enfrentam os problemas da limitação técnica do tamanho.

Já outras teorias heterodoxas avançaram nas últimas décadas no estudo da competitividade das empresas menores, bem como na análise dos fatores econômicos e não econômicos para a viabilidade destas empresas. 0 destaque da cooperação e da aglomeração produtiva está presente nesta literatura, que demonstra a necessidade de instrumentos de desenvolvimento regional para as pequenas empresas. A empresa não é analisada de forma isolada, mas sim formando um mosaico produtivo com outras empresas da mesma região, que é especializada e inovadora. A vantagem reside na aproximação da empresa e pouco se analisa a sua situação particular.

A limitação da análise microeconômica da uma MPE reside na sua baixa escala e falta de gestão. Na maioria das vezes o próprio empresário ou um familiar controla todas as principais tarefas da empresa, como o controle financeiro, de custos ou o relacionamento com clientes e fornecedores. A baixa formação dos proprietários limita a capacidade de aprendizado, de inovação e de adoção de boas práticas de gestão nestas empresas, o que explica a alta taxa de mortalidade das empresas nesta faixa de tamanho.

Aqui se apresenta um programa diferenciado, em escala nacional e voltado para as necessidades das MPEs: o programa Agentes Locais de Inovação (ALI) do SEBRAE em parceria com o CNPQ. Este programa utiliza uma metodologia inovadora no estudo das MPEs no Brasil, baseada no Radar da Inovação.

Este artigo analisa os resultados da aplicação do Radar da Inovação em todas as empresas participantes do programa ALI em Santa Catarina no ciclo 
2015-2018. O total de empresas é de 3.675 em todos os setores econômicos, onde 464 são microempresas e 3.211 são de pequeno porte.

Os resultados analisados estão relacionados com a avaliação inicial e o primeiro ciclo. Mais dois ciclos ainda serão produzidos, o que torna estes resultados ainda preliminares, mas importantes para se apresentar a importância do Radar da Inovação como um instrumento de análise do grau de inovação nas MPEs e para se testar a hipótese de homogeneidade das empresas. Esta hipótese tem o objetivo de verificar se existem diferenças ou não micro e pequenas empresas, já que a literatura não apresenta diferenças entre estas empresas em função de seu tamanho, o que conduz a ideia de que elas são iguais, com os mesmos limites e desafios. Como os dados do programa ALI em Santa Catarina são agrupados pelo tamanho da empresa, há como se verificar o desempenho das micro e das pequenas empresas. Desta comparação pode-se verifica se as mesmas apresentam diferenças nas dimensões analisadas.

Este artigo é composto de cinco seções, contando com esta introdução. A seção dois apresenta a identificação na literatura da importância das MPEs no mercado, seus desafios e perspectivas de políticas públicas de incentivo, bem como analisa a relação entre as empresas de pequeno porte e a inovação, esta seção apresenta uma revisão da literatura internacional e nacional, A seção três apresenta a fonte de dados utilizada e o programa ALI. A seção quatro analisa os resultados encontrados e por fim, a seção cinco conclui $o$ artigo.

\section{A IMPORTÂNCIA DA MICRO E PEQUENA EMPRESA}

$\mathrm{Na}$ indústria dos países da OCDE a produção industrial migrou das grandes para as pequenas empresas nas últimas duas décadas (anos 70 e 80 ), o que possibilitou o desenvolvimento de políticas públicas incentivando as pequenas empresas na Europa na década de 80 em resposta ao ambiente de incertezas com a mudança tecnológica (CARRE e THURIK 1998).

A análise dos resultados destas políticas públicas mostra que os países com maior participação de empresas pequenas crescem mais, ou seja, as políticas de incentivo as pequenas empresas aceleraram a economia. Mas para tanto, torna-se necessário discutir a escala, escopo, experiência e dependência nas pequenas empresas, em função das pequenas empresas serem mais intensivas em trabalho.

Cooke e Wills (1999) mostram que a pequenas empresas crescem com a formação de capital social, que é mensurado em termos de número, intensidade, interdependência, contatos, redes e engajamento civil. Portanto, as políticas públicas para criar colaboração (capital social) nas empresas, devem considerar com destaque o papel do território, aumentando o desempenho, a exploração do conhecimento e a inovação nas pequenas empresas.

Desta forma, para crescer as empresas necessitam formar capital social, verificado no papel central do território nas pequenas empresas. No território as empresas interagem com as instituições que promovem o 
crescimento, inovação, o que evita a alta mortalidade destas empresas. Instituições como as universidades, governo (local e nas demais esferas), demais empresas da cadeia produtiva (fornecedores especializados, consumidores), mão de obra qualificada e toda a rede de estrutura e incentivos presentes na aglomeração territorial são importantes e essenciais para o sucesso de qualquer política pública que estimule as pequenas empresas.

Percebe-se que as pequenas empresas enfrentam barreiras estruturais para inovarem. A hipótese de Schumpeter é de que as empresas maiores inovam mais, ou seja, existe competição imperfeita para inovar (ACS e AUDRETSCH 1987). Todavia, existem mercados em que as empresas menores inovam mais, que são mercados com maior competição, já as empresas maiores são favorecidas em mercados com menor competição.

\subsection{REVISÃO DA LITERATURA INTERNACIONAL}

A literatura internacional analisa como as MPEs podem ser mais eficientes e lucrativas ao se inserirem em sistemas de inovação aberta (CHOI el al. 2016; SAGUY e SIROTINSKAYA 2014).

A aglomeração produtiva também é incentivada. Estudos como Chen et al. (2017) mostram como as MPEs são mais produtivas quando se aproximam em sistemas locais de produção, ou a formação de redes associativas (QIAO et al. 2014). Aglomerações regionais inovativas também são apresentadas em estudos como Herliana (2015).

O desenvolvimento sustentável como resposta aos problemas ambientais atuais é um ponto de destaque da literatura sobre MPEs. O estudo de Chen et al. (2017) mostra como a poluição limpa, em ecoparques pode beneficiar as MPEs, o que Saéz-Martinez et al. (2016) denomina de ecoinovação. As bases do desenvolvimento sustentável podem beneficiar as empresas menores, mais flexíveis e adaptadas ao meio (FELLNHOFER 2017).

Por fim, a literatura discute o papel positivo das inovações sobre os pequenos negócios (WOSCHKE e WAASE 2016; FORSMAN 2011; SOLTÉS e GAVUROVÁ 2014; OKWIET e GRABARA 2013; MAZZEI et al. 2016; SKIBÍNSKI e SIPA 2015; OSENIEKS e BABAUSKA 2014). A estratégia de adoção de inovações nas MPEs está relacionada com a adoção de políticas públicas específicas voltadas para o porte menor destas empresas, bem como o incentivo ao empreendedorismo (ROMERO e MARTÍNEZ-ROMÁN 2012; DUNNE et al. 2016; YANG et al. 2017). De forma específica Lisowska e Stanislawski (2015) mostram como o sistema de inovação aberto pode beneficiar as MPEs, a inovação passa a ser colaborativa e não mais competitiva para as empresas inovadoras neste contexto.

\subsection{A MICRO E PEQUENA EMPRESA NO BRASIL}

A literatura brasileira sobre as MPEs analisa a vantagem alocativa destas empresas em sistemas de produção. Parte-se do princípio, facilmente observado, de que as pequenas empresas apresentam falta de 
competitividade, baixo dinamismo tecnológico e pouca ou quase nada de inovação. Neste sentido, a solução é a cooperação.

Os principais sistemas de produção apontados são os Arranjos Produtivos Locais, denominados de APLs (NARETTO et al. 2004;). A formação de clusters também é identificada na literatura nacional, onde o cluster é a aglomeração local de empresas, sem a formação de um sistema de coordenação organizado, estudos como Cezarino e Campomar (2005) e Rovere e Shehata (2006) mostram a contribuição desta forma de aglomeração geográfica no desempenho das MPEs.

Estratégias microeconômicas também são identificadas para as MPEs. Silva e Dacorso (2013) analisam o impacto diferenciado e positivo da inovação aberta nestas empresas. Já o estudo de Lima e Mendes (2003) mostra a importância da gestão organizacional para as MPEs inovarem e promoverem a mudança tecnológica. O perfil inovador do empreendedor é destacado no estudo de Sarfati (2013), para o autor o empreendedorismo é um fator chave para o crescimento das pequenas empresas.

Por fim, a inovação é apresentada como um importante fator para o sucesso das pequenas empresas. O estudo de Claudino et al. (2017) identifica os fatores que limitam a capacidade de inovação nestas empresas. Já o impacto positivo da inovação pode ser visto nos estudos de Aksoy (2017) e Silva et al. (2016), onde as inovações são mais percebidas com a adoção de políticas públicas voltadas para a realidade das MPEs, como o programa ALI, como argumenta Lima e Müller (2017).

\section{METODOLOGIA E FONTE DE DADOS}

A metodologia do Radar da Inovação foi adaptada para o SEBRAE no estudo de Bachman e Associados (2008) para utilização no programa Agentes Locais de Inovação, que é uma parceria entre o SEBRAE nacional com os SEBRAEs estaduais e CNPQ. A ideia do Radar da Inovação é mensurar a cultura da inovação na empresa, através de uma métrica nos pontos relevantes para uma empresa de pequeno porte inovar. As variáveis analisadas pelo Radar são: 1) Oferta, 2) Plataforma, 3) Marca, 4) Clientes, 5) Soluções, 5) Relacionamento, 6) Agregação de Valor, 7) Processos, 8) Organização, 9) Cadeia de Fornecimento, 10) Presença, 11) Rede, 12) Ambiência Inovadora. O quadro 1 a seguir resume as dimensões do Radar da Inovação e que são o objeto de análise deste estudo.

Quadro 1: Dimensões do Radar da Inovação

\begin{tabular}{|l|l|}
\hline \multicolumn{1}{|c|}{ Dimensão } & \multicolumn{1}{|c|}{ Interpretação } \\
\hline Oferta & $\begin{array}{l}\text { Inovações em produtos oferecidos no } \\
\text { mercado, avaliando a disposição da } \\
\text { empresa em assumir riscos e ousadia. }\end{array}$ \\
\hline Plataforma & $\begin{array}{l}\text { Forma como a empresa utiliza os seus } \\
\text { recursos de modo a oferecer maior } \\
\text { variedade de produtos. }\end{array}$ \\
\hline Marca & Como a empresa protege e utiliza a sua \\
\hline
\end{tabular}




\begin{tabular}{|l|l|}
\hline & $\begin{array}{l}\text { marca para promover oportunidades de } \\
\text { negócio. }\end{array}$ \\
\hline Clientes & $\begin{array}{l}\text { Avalia coo a empresa busca entender as } \\
\text { necessidades de seus clientes. }\end{array}$ \\
\hline Soluções & $\begin{array}{l}\text { Oferta de produtos e serviços } \\
\text { complementares oferecidos aos clientes. }\end{array}$ \\
\hline Relacionamento & $\begin{array}{l}\text { Ações para promover interações positivas } \\
\text { nos pontos de contatos com os clientes. }\end{array}$ \\
\hline Agregação de valor & $\begin{array}{l}\text { Explorar o fluxo de receitas para gerar } \\
\text { novas fontes de receitas. }\end{array}$ \\
\hline Processos & $\begin{array}{l}\text { Ações realizadas para aumentar a } \\
\text { eficiência, qualidade ou produtividade. }\end{array}$ \\
\hline Organização & $\begin{array}{l}\text { Formas de estruturação da empresa, do } \\
\text { papel dos colaboradores e de parcerias } \\
\text { com o intuito de formar alterações } \\
\text { estratégicas. }\end{array}$ \\
\hline Cadeia de fornecimento & $\begin{array}{l}\text { Aspectos logísticos do processo e busca } \\
\text { por redução de custos. }\end{array}$ \\
\hline Presença & $\begin{array}{l}\text { Abertura de novos canais de venda } \\
\text { diferentes dos usuais. }\end{array}$ \\
\hline Rede & $\begin{array}{l}\text { Recurso utilizados pela empresa para se } \\
\text { comunicar de modo eficaz com seus } \\
\text { clientes. }\end{array}$ \\
\hline Ambiência inovadora & $\begin{array}{l}\text { quanto o ambiente é propício à } \\
\text { inovação. Considera as fontes de } \\
\text { informação acessadas, depósitos de } \\
\text { patentes, mecanismos de sugestão e } \\
\text { acesso a fones de financiamento para } \\
\text { inovar. }\end{array}$ \\
\hline
\end{tabular}

Fonte: adaptado de Bachmann \& Associados (2008).

O Objetivo das dimensões é identificar os fatores relacionados com a capacidade organizacional e de relacionamento com o mercado nas empresas. Estas características são essenciais para a diferenciação e a inovação das empresas. Atualmente, não há espaço para ações empresariais sem conhecer o seu ambiente.

As empresas são identificadas e pertencem a vários setores da economia, não há distinção entre os setores. Cada agente se limita a uma região do estado, ao todo o programa conta com quarenta agentes em campo atuando em todos os setores e cobrindo o estado todo.

Após a adesão ao programa, que é voluntária, o empresário faz uma avaliação das dimensões do Radar. Com este diagnóstico em mãos, o ALI faz um acompanhamento, sugerindo ações que impulsionem a inovação na empresa, estas ações são acompanhadas pelo ALI ao longo do período do projeto. O problema identificado pelo SEBRAE é a falta de capacidade de gestão nas empresas de pequeno porte, o que impede o seu sucesso e inviabiliza a adoção de inovações, conduzindo grande parte destas empresas ao fechamento precoce.

As métricas utilizadas variam em três: i) zero (a empresa não inovou), ii) três ( a empresa inovou esporadicamente) e iii) cinco (a empresa é inovadora). Aqui a mensuração não está preocupada com inovações radicais, que são novidades no mercado, mas sim, busca identificar inovações em 
atitudes simples, que diferenciem os produtos e serviços da empresa, gerando mais receita e contribuindo com o sucesso da empresa. Em resumo, o Radar busca mensurar a cultura inovadora, que pode estar na forma de inovações em produto, processo, organizacionais ou mercadológicas.

Em relação aos valores, as variáveis são discretas ao assumirem três possíveis valores. Porém, como o Projeto ALI é de dois anos, pelo menos três rodadas do Radar são realizadas e os valores finais são valores médios que podem assumir qualquer valor entre zero e cinco. Os resultados apurados são apresentados na próxima seção.

\section{RESULTADOS}

Os valores apurados para o conjunto de variáveis $i$ do $\operatorname{Radar}\left(\boldsymbol{V}_{i}\right)$ para as empresas participantes do programa ALI estão apresentados nos gráficos 1 e 2. Os valores são médios e dizem respeito aos ciclos zero (0) e um (1) do Radar e estão definidos no domínio entre $0 \leq \boldsymbol{V}_{i} \leq 5$.

Figura 1: Média do Radar para o ciclo zero

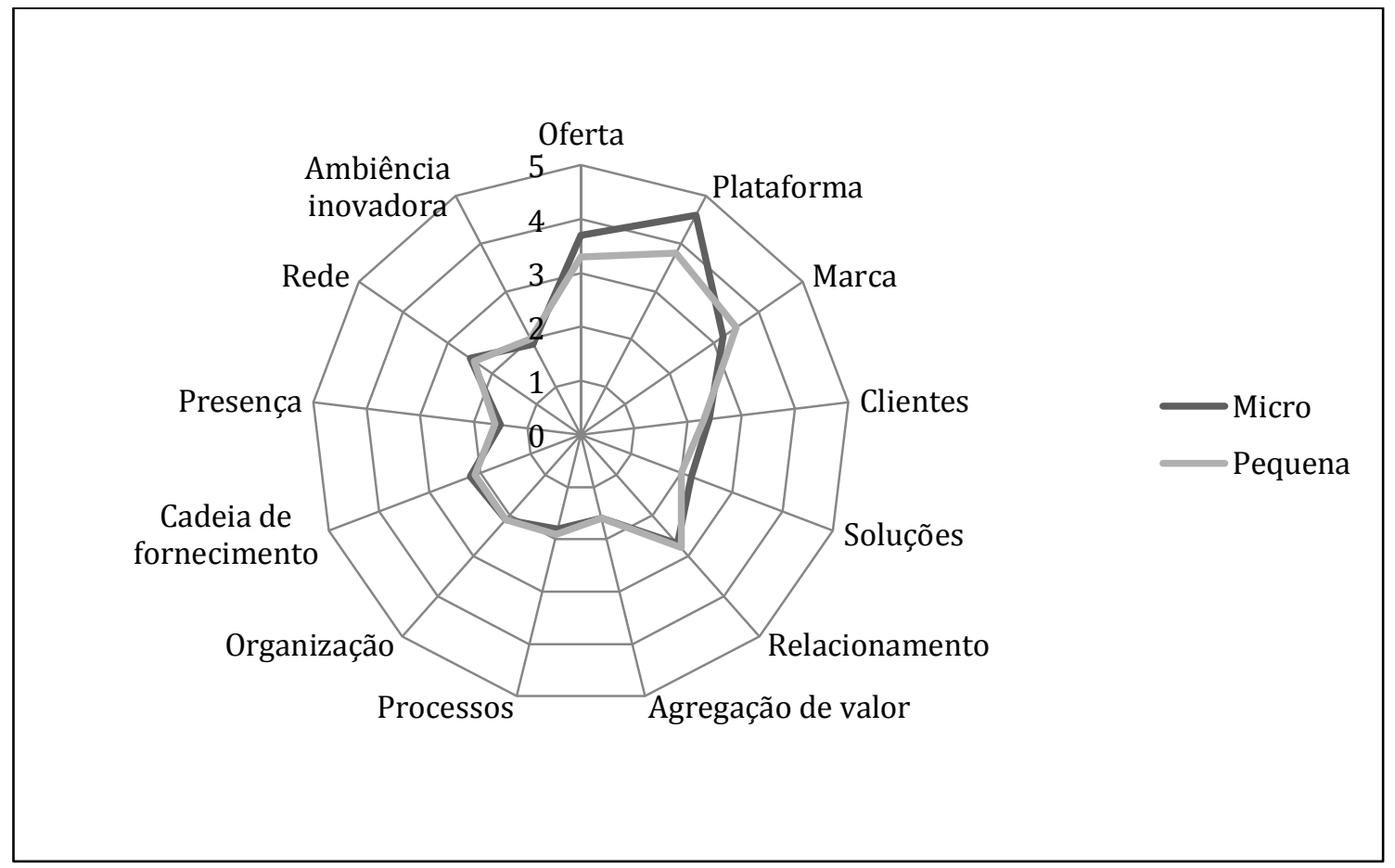

Fonte: O Autor com dados do SystemALI

Os dados no gráfico 1 mostram que para as dimensões do Radar existem poucas diferenças entre as micro e pequenas empresas em relação às dimensões analisadas. Apenas três dimensões apresentaram diferenças significativas. As dimensões Oferta e Plataforma apresentam valores maiores para as microempresas em relação às empresas de pequeno porte. A dimensão Marca é maior para as pequenas empresas em relação às microempresas.

Os resultados para o primeiro ciclo estão no gráfico dois. 
Figura 2: Média do Radar para o ciclo um

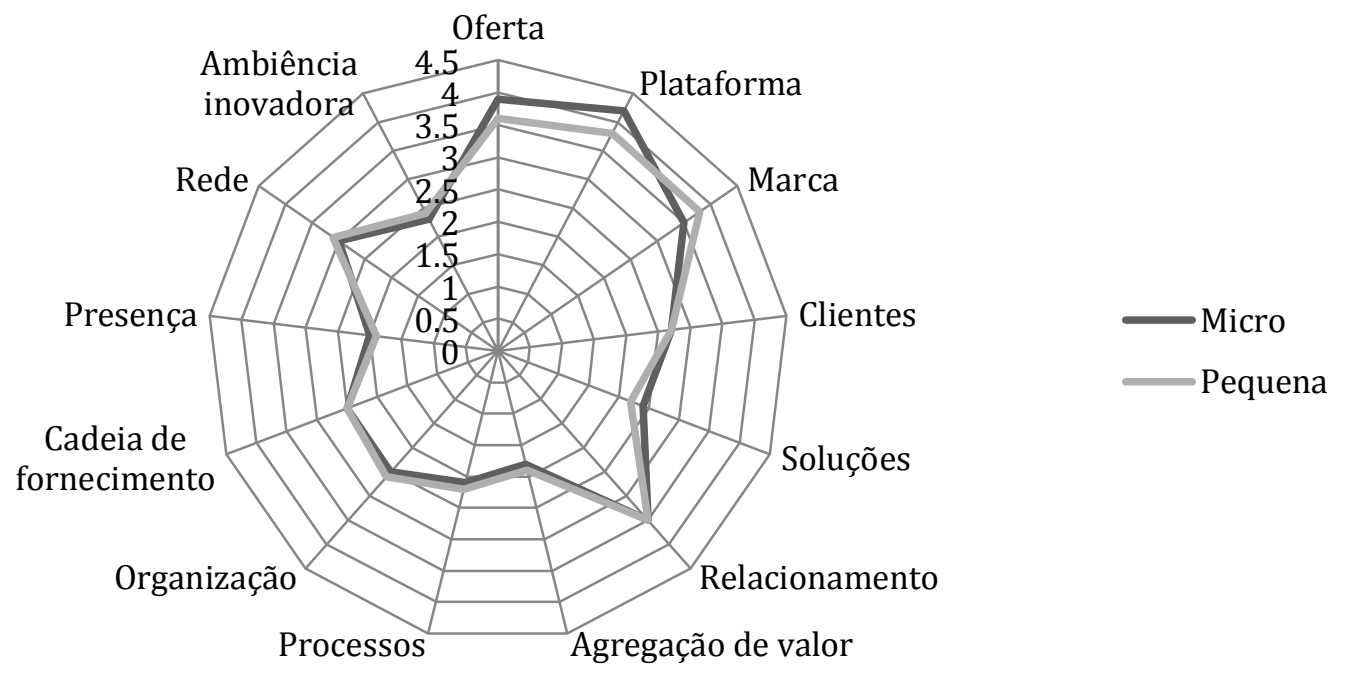

Fonte: O Autor com dados do SystemALI

Os dados apresentados no gráfico dois mostram o aumento dos indicadores do ciclo inicial (zero) para o ciclo 1. O crescimento manteve as mesmas diferenças e semelhanças entre as dimensões. As taxas de crescimento das dimensões do ciclo zero para o ciclo um são apresentadas na tabela um a seguir para os dois tamanhos de empresa.

Tabela 1: Taxas de crescimento do ciclo zero para o ciclo um percentual

\begin{tabular}{lcc}
\hline \multicolumn{1}{c}{ Dimensão } & Micro & Pequena \\
\hline Oferta & $5,41 \%$ & $9,09 \%$ \\
Plataforma & $-8,70 \%$ & $0,00 \%$ \\
Marca & $9,37 \%$ & $8,57 \%$ \\
Clientes & $12,50 \%$ & $17,39 \%$ \\
Soluções & $9,09 \%$ & $10,00 \%$ \\
Relacionamento & $29,63 \%$ & $25,00 \%$ \\
Agregação de valor & $12,50 \%$ & $18,75 \%$ \\
Processos & $16,67 \%$ & $15,79 \%$ \\
Organização & $19,05 \%$ & $23,81 \%$ \\
Cadeia de fornecimento & $13,64 \%$ & $19,05 \%$ \\
Presença & $33,33 \%$ & $18,75 \%$ \\
Rede & $20,00 \%$ & $29,17 \%$ \\
\hline
\end{tabular}

Fonte: O Autor com dados do SystemALI

Os dados mostram que as dimensões Rede e Relacionamento, que são muito próximas, apresentaram as maiores taxas de crescimento para as micro 
e pequenas empresas, sempre acima de 20\%. Outros destaques foram o crescimento de $33,33 \%$ para a dimensão Presença e de $20 \%$ para a dimensão Rede nas microempresas. As pequenas empresas apresentaram as maiores taxas de crescimento nas dimensões, além de Rede e Relacionamento, a dimensão Organização, com $23,81 \%$ de crescimento. Estes resultados mostram que as micro e pequenas empresas apresentam maior destaque estratégico nas dimensões relacionadas com o mercado

As dimensões que se destacaram com as menores taxas de crescimento foram Oferta, Plataforma, Marca e Soluções, sempre com valores menores que $10 \%$. Estes resultados mostram que as micro e pequenas empresas enfrentam dificuldades nas dimensões relacionadas com a identidade da empresa.

Além da taxa de crescimento e da análise gráfica, a tabela seguinte mostra dois testes estatísticos realizados para comparar os valores das micro e das pequenas empresas nos indicadores selecionados para os ciclos zero e um do Radar.

Tabela 2: Testes $t$ e $F$ para as dimensões do radar nos ciclos 0 e 1

\begin{tabular}{|c|c|c|c|c|c|}
\hline & & \multicolumn{2}{|c|}{ Teste $t$} & \multicolumn{2}{|c|}{ Teste $F$} \\
\hline & & Micro & Pequenas & Micro & Pequenas \\
\hline$\frac{\bar{\pi}}{\frac{\pi}{\pi}} \underset{\alpha}{\alpha}$ & $\begin{array}{l}\text { Média } \\
\text { Variância } \\
\text { Valor }(t, F) \\
\text { Significância }(\rho)\end{array}$ & $\begin{array}{l}2,49 \\
0,78 \\
1,00 \\
0,16 \\
\end{array}$ & $\begin{array}{l}2,41 \\
0,51\end{array}$ & $\begin{array}{l}2,8 \\
0,57 \\
2,68 \\
0,38 \\
\end{array}$ & $\begin{array}{l}2,75 \\
0,485\end{array}$ \\
\hline$\frac{\frac{1}{\pi}}{\frac{\pi}{\pi}}-1$ & $\begin{array}{l}\text { Média } \\
\text { Variância } \\
\text { Valor }(t, F) \\
\text { Significância }(\rho)\end{array}$ & $\begin{array}{l}2,8 \\
0,57 \\
0,29 \\
0,38 \\
\end{array}$ & $\begin{array}{l}2,78 \\
0,488\end{array}$ & $\begin{array}{l}3,02 \\
0,51 \\
2,68 \\
0,404\end{array}$ & $\begin{array}{l}3,13 \\
0,44\end{array}$ \\
\hline
\end{tabular}

$t$ crítico de 1,78 para $5 \%$.

Fonte: O Autor com dados do SystemALI.

Os resultados de ambos os testes confirmam que as médias dos dois grupos são iguais. Os valores apresentam grau de significância maior que o valor crítica de 5\%, o que não permite a rejeição da hipótese nula de que as médias das micro e das pequenas empresas sejam iguais.

Percebe-se que as micro e pequenas empresas catarinenses participantes do programa Agentes Locais de Inovação não diferem nos resultados do Radar da Inovação. Neste sentido, a percepção dos empresários frente aos desafios encontrados e a definição de estratégias e ações pertinentes são semelhantes nos dois tamanhos de empresas.

\section{CONCLUSÃO}

Este artigo analisou os resultados das empresas participantes do programa Agentes Locais de Inovação do SEBRAE Santa Catarina com apoio do CNPQ. Os dados mostram pouca diferença entre o desempenho das micro e das pequenas empresas, neste sentido, conclui-se que os dois tamanhos de 
empresa de pequeno porte apresentam o mesmo comportamento em relação á inovação. Os limites e dificuldades encontrados são análogos, assim como os benefícios.

O teste estatístico de equivalência das médias entre os dois grupos de empresas, a análise gráfica e a taxa de variação entre os indicadores mostraram que não existem diferenças entre os dois tamanhos de empresa em relação ao desempenho nos indicadores de inovação do Radar utilizado pelo programa ALI do SEBRAE-SC.

Este resultado ajuda na compreensão da análise em conjunto das empresas de pequeno porte. A teoria, as análises e resultados sempre colocam estas empresas na mesma faixa de tamanho. Os dados levantados pelo programa ALISC permitem a diferenciação de duas faixas de tamanho, todavia, os resultados mostram que as empresas apresentam resultados muito semelhantes.

Em relação ao impacto do programa sobre as empresas analisadas, percebe-se que as dimensões relacionadas às estratégias de relacionamento da empresa com o mercado apresentaram taxas de crescimento maior, o que mostra que as MPEs participantes do programa seguiram uma estratégia de se aproximar mais do mercado em relação às demais dimensões. Este resultado mostra que as empresas de pequeno porte buscam aproximação com o seu cliente e com a rede de colaboradores, como fornecedores.

Para efeito de políticas públicas, este resultado mostra que as empresas de pequeno porte sentem que as ações de aproximação com o mercado surtem mais efeitos. Estratégias como a participação em feiras, eventos, bem como a ações que mostram mais a empresa e sua visualização são consideradas mais eficientes, o que mostra que os empresários se sentem isolados e desconhecidos. Nesta direção, explorar as novas ferramentas digitais pode ser uma estratégia simples, direta e que aproxime a MPE de novos clientes.

\section{REFERÊNCIAS}

ACS, Z.J.; AUDRETSCH, D.B. Innovation, market structure, and firm size. The Review of Economics and Statistics, volume 69, number 4, p. 567-574, 1987.

AKSOY, H. How do innovation culture, marketing innovation and product innovation affect the market performance of small and medium-sized enterprises (SMEs)? Technology in Society, volume 51, p. 133-141, 2017.

BACHMAN \& ASSOCIADOS. Metodologia para estimar o grau de inovação nas MPE. Curitiba: Bachman \& Associados, 2008.

CARRE, M.A.; THURIK, A.R. Small firms and economics growth in Europe. American Economic Review, volume 26, number 2, p. 137-146, 1998.

CEZARINO, L. O.; CAMPOMAR, M. C. Vantagem competitiva para a micro, pequenas e médias empresas: clusters e APLS. Curitiba: Anais do Encontro de 
Estudos sobre Empreendedorismo e Gestão de Pequenas Empresas, p. 13151326, 2005.

CHEN, L.; ZHOU, Z.; ZHOU, D. ; XUE, L. Clustering enterprises into eco-industrial parks: can interfirm alliances help small and medium-size enterprises? Journal of Cleaner Production, volume 168, p. 1070-1079, 2017.

CHOI, B.; LEE, J. N.; HAM, J. Assessing the impact of open and close knowledge sourcing approach on innovation in small and medium enterprises. Procedia Computer Sciences, volume 91, p. 314-323, 2016.

CLAUDINO, T. B.; SANTOS, S. M.; CABRAL, A. C. A.; PESSOA, M. N. M. Fostering and limiting factors of innovation in micro and small enterprises. Innovationa and management review, volume 14, p. 130-139, 2017.

COOKE, P.; WILLS, D. Small firms, capital social and the enhancement of business performance through innovation. Small Business Economics, volume 13, p. 219-234, 1999.

DUNNE, T. C.; AARON, J. R.; MCDOWELL, W. C.; URBAN, D. J.; GEHO, P. R. The impact of leadership on small business innovativeness. Journal of Business Research, volume 69, p. 4876-4881, 2016.

FELLNHOFER, K. Drivers of innovation success in sustainable business. Journal of Cleaner Production, volume 167, p. 1534-1545, 2007.

FORSMAN, $\mathrm{H}$. Innovation capacity and innovation development in small enterprises. A comparison between the manufacturing and service sectors. Research Policy, volume 40, p. 739-750, 2011.

HERLIANA, S. Regional innovation cluster of small and medium enterprises (SME): a triple helix concept. Procedia Social and Behavioral Sciences, volume 169 , p. 151-160, 2015.

LIMA, M. A. M.; MENDES, J. P. F. Inovação na gestão organizacional e tecnológica: conceitos, evolução histórica e implicações para as micro, pequenas e médias empresas no Brasil. Revista Produção, volume 3, número 2, p. 1-23, 2003.

LIMA, V. A.; MÜLLER, C. A. S. Why do small business innovate? Relevance factors of innovation in business participating in the local agents program in Rondônia (Amazon, Brazil). Revista de Administração e Inovação, no prelo, 2017.

LISOWSKA, R.; STANISLAWSKI, R. The cooperation of small and medium-sized enterprises with business institutions in the context of open innovation. Procedia Economics and Finance, volume 23, p. 1273-1278, 2015.

MAZZEI, M. J.; FLYNN, C. B.; HAYNIE, J. J. Moving beyond initial success: promoting innovation in small businesses through high-performance work practices. Business Horizons, volume 59, p. 51-60, 2016. 
NARETTO, N.; BOTELHO, M. dos R.; MENDONÇA, M. A trajetória das políticas públicas para pequenas e médias empresas no Brasil: do apoio individual ao apoio a empresas articuladas em arranjos produtivos locais. Planejamento $e$ Políticas Públicas, volume 27, p. 61-115, 2004.

OKWIET, B.; GRABACA, J. K. Innovation' influences on SMEs's enterprises activities. Procedia Economics and Finance, volume 6, p. 194-204, 2013.

OSENICKS, J.; BABAUSKA, $\mathrm{S}$. The relevance of innovation management as prerequisite for durable existence of small and medium enterprises. Procedia Social and Behavioral Sciences, volume 110, p. 82-92, 2014.

QIAO, P. H.; JU, X. F.; FUNG, H. G. Industry association networks, innovations, and firm performance in Chinese small and medium-sized enterprises. Chinese Economic Review, volume 29, p. 213-228, 2014.

ROMERO, I.; MARTÍNEZ-ROMÁN, J. A. Self-employment and innovation. Exploring the determinants of innovative behavior in small businesses. Research Policy, volume 41, p. 178-189, 2012.

ROVERE, R. L. la; SHEHATA, L. D. Políticas de apoio à micro e pequenas empresas e desenvolvimento local: alguns pontos de reflexão. Redes, volume 11, número 3, p. 9-24, 2006.

SAGUY, S.; SIROTINSKAYA, V. Challenges in exploiting open innovation's full potential in the food industry with a focus on small and medium enterprises (SMEs). Trends in Food Science \& Technology, volume 38, p. 136-149, 2014.

SAÉZ-MARTÍNEZ, F. J.; DÍAZ-GARCÍA， C.; GONZALEZ-MORENO, A. Firm technological trajectory as a driver of eco-innovation young small and mediumsized enterprises. Journal of Cleaner Production, volume 138, p. 28-37, 2016.

SARFATI, G. Estágios de desenvolvimento econômico e políticas públicas de empreendedorismo e de micro, pequenas e médias empresas (MPMEs) em perspectiva comparada: os casos do Brasil, do Canadá, do Chile, da Irlanda e da Itália. Revista de Administração Pública, volume 47, número 1, p. 25-48, 2013.

SILVA, F. M.; OLIVEIRA, E. A. A. Q.; MORAES, M. B. Innovation development process in small and medium technology-based companies. Revista de Administração e Inovação, volume 13, p. 176-189, 2016.

SILVA, G.; DACORSO, A. L. R. Inovação aberta como uma vantagem competitiva para a micro e pequena empresa. Revista de Administração e Inovação, volume 10, número 3, p. 251-268, 2013.

SKYBÍNSKY, A.; SIPA, M. Sources of innovation of small businesses: Polish perspectives. Procedia Economics and Finance, volume 27, p. 429-437, 2015.

SOLTÉS, V.; GAVUROVÁ, B. Innovation policy as the main accelerator of increasing the competitiveness of small and medium-sized enterprises in Slovakia. Procedia Economics and Finance, volume 15, p. 1478-1485, 2014. 
WOSCHKE, T.; HAASE, H. Enhancing new product development capabilities in small-and-medium-size enterprises through managerial innovations. Journal of High Technology Management Research, volume 27, p. 53-64, 2016.

YANG, M.; EVANS, S.; VLADIMIROVA, D.; RANA, P. Value uncaptured perspective for sustainable business model innovation. Journal of Cleaner Production, volume 140, p. 1794-1804, 2017. 